\title{
Género, política e hibridismo en la transnacionalización de la cultura Yoruba*
}

Rita Segato

\section{Resumo}

O artigo examina três interpretações diferentes da construção de gênero na tradição Yoruba e as analisa enquanto discursos posicionados e interessados dos autores no contexto de seus respectivos horizontes políticos de referência. Há, neste sentido, uma verdadeira economia política do discurso etnográfico. Contudo, constata que os três autores, apesar das suas diferenças, apontam de forma inconfundível para o caráter radicalmente anti-essecialista das concepções de gênero Yoruba. Sugere, então, que essa característica tão notável desta tradição poderia estar na base da sua capacidade de expansão e adaptação no Novo Mundo, tanto no período pós-escravidão como no novo período de expansão que vivem atualmente as religióes afro-brasileiras de origem Yoruba.

Palavras-chave: Diáspora Yoruba, gênero, discurso etnográfico.

* Este texto, bajo el título The Gender Factor in the Yoruba Transnational Religious World, fue presentado en sesión plenaria durante la Conferencia Internacional de Académicos de las Ciencias Sociales en Estudios de Religión ocurrida en Houston, Texas, en octubre de 2000. 
Rita Segato

\begin{abstract}
Gender, politics and hybridism in the transnationalization of the Yoruba culture
\end{abstract}

The article examines three different interpretations over the construction of gender in the Yoruba tradition and analyzes them as autors' based and biased speeches in the context of their respective political horizons. There is a real economical politics in the ethnographic speech. It shows that the three authors, in spite of their differences, incomparably highlight the radically anti-essentialist conception of the Yoruba gender. It suggests that this tradition's noticeable characteristic could be the basis of its capacity of expansion and adaptability in the New World, during the post-slavery period and also during the expansionist period that those African-Brazilian religions originated from Yoruba have been going through nowadays.

Keywords: Yoruba exodus, gender, ethnographic speech.

\title{
Résumé
}

Genre, politique et hybridisme dans la transnationalisation de la culture Yoruba

Dans cet article, on examine trois interprétations différentes de la construction du genre dans la tradition Yoruba en tant que discours où les auteurs prennent position et s'intéressent au contexte de leurs horizonts politiques de référence. Il y a, dans ce sens, une véritable économie politique du discours ethnographique. Les tois auteurs, malgré leurs différences, montrent l'aspect radicalement antiessencialiste de la conception du genre Yoruba. Ils proposent donc que cette caractéristique si remarquable de cette tradition pourrait être à la base de sa capacité d'éxpansion et d'adaptation dans le Nouveau Monde; cela serait valable tant pour la période post-esclavagiste que pour la nouvelle période d'expansion actuelle des religions afro-brésiliennes d'origine Yoruba.

Mots-clés: diaspora Yoruba, genre, discours ethnographique. 
Las teorías antropológicas de modo general hablan más sobre los antropólogos de que sobre su disciplina. (Edmund Leach 1966).

... all knowledge of other cultures, societies, or religions comes about through an admixture of indirect evidence with the individual scholar's personal situation, which includes time, place, personal gifts, historical situation, as well as the overall political circumstances. What makes such knowledge accurate or inaccurate, bad, better, or worse, has to do mainly with the needs of the society in which that knowledge is produced. (Edward W. Said, 1997:168)

$D$ resento aquí tres diferentes discursos académicos que abordan las ideas de género de la civilización Yoruba y, a pesar de sus diferencias, vinculo sus hallazgos a dos momentos históricos de la expansión de esa cultura distantes en el tiempo y en las circunstancias. Conduzco la comprensión de los modelos interpretativos que presentan para lo que entiendo como el factor género en la difusión de la cosmología Yoruba. En la primera parte del texto caracterizo sintéticamente esos tres discursos académicos, mostrando cómo, a pesar de sus diferencias, luchan con las palabras disponibles por describir, en etnografías de extrema complejidad, la sofisticada y muy peculiar concepción de género en el universo de la cultura Yoruba. Al hacerlo, proponen modelos diferentes, pero acaban dejando de manifiesto que apuntan para una realidad común: el alto grado de abstracción de la construcción de género en relación con los significantes anatómicos, es decir, la ausencia de esencialismo biológico que el sistema de pensamiento presenta. En la segunda parte del texto, analizo cómo los discursos académicos se encuentran nacional e interesadamente situados y muestro que, de modo bastante independiente, los tres autores, yo misma entre ellos, colocaron los complejos temas de género en el centro del debate sobre la visión del mundo Yoruba. Retomo allí suscinta- 
mente lo que escribieron para tratar de revelar cómo la posición (étnica y nacional) a partir de la cual cada autor produce sus modelos afecta sus formulaciones. En la tercera parte del texto, someto a una breve revisión mis propias ideas y sostengo que las complejidades de la construcción de género Yoruba fueron centrales en el proceso de difusión de la religión Yoruba. Hay evidencias de que los bloques de contexto social que ese proceso de difusión diseminó de Africa para el Nuevo Mundo inicialmente y, posteriormente, de Brasil para nuevos países donde su influencia no existía como Argentina y Uruguay, contienen siempre los elementos de un ideario de género no esencialista, que permitió y permite la maleabilidad adaptativa de las relaciones familiares, sexuales y afectivas en las condiciones severísimas que los afro-descendientes tuvieron que soportar.

\section{Tres antropólogos en el debate sobre el género en el mundo religioso yoruba}

Haré referencia aquí a tres modelos interpretativos de las ideas Yoruba de género, tal como se expresan en los temas y en las prácticas religiosas. Los dos que tratan sobre los Yoruba de Nigeria fueron formulados por Lorand Matory, en 1994, y Oyeronke Oyewumi, en 1997, respectivamente, y ambos publicados por la prestigiosa editora de la Universidad de Minnesota. El tercer modelo proviene de mi propio trabajo sobre la religión Yoruba en Brasil, publicado en portugués en 1986 (y reimpreso en esa lengua en 1989, 1995 y 2000, como también publicado en inglés en 1997). Aunque mi publicación sobre el tema es anterior a la de los otros dos autores, invierto aquí el orden temporal e inicio la discusión por el trabajo de Oyeronke para conseguir mayor claridad en el argumento.

\section{Oyeronke Oyewumi}

Oyeronke, Yoruba ella misma, publicó The Invention of Women. Making an African Sense of Western Gender Discourses cuando era profesora-asistente en la Universidad de California, en Santa Bárbara, Estados Unidos. A pesar de estar trabajando en el mismo campo, Oyeronke no hizo allí referencia al libro de Matory, de 1994, sobre tópico similar, citando apenas, y en dos párrafos que 
no exceden la extensión de una página, la tesis de maestría del autor, presentada en 1991.

Para Oyeronke, "la hipótesis de que un sistema de género existía en la sociedad Oyo con anterioridad a la colonización occidental constituye un caso más de la dominación occidental en la documentación y en la interpretación del mundo" (1997:32). Desde su punto de vista, el colonialismo introdujo el vocabulario y las prácticas de género en la religión Yoruba, y los académicos occidentales -así como las feministas occidentales- equivocadamente identificaron la existencia del género en esa cultura: "[...] la interpretación usual de las categorías Yoruba obinrin y okunrin como 'hembra/mujer' y 'macho/hombre', respectivamente, es una traducción equivocada [...] porque (entre los Yoruba pre-coloniales) esas categorías no se oponían binariamente ni eran jerárquicas [...]" (ibidem: 32-33). Eso es así, en la interpretación de Oyeronke, porque, como ella misma argumenta:

1) "No existía ahí la concepción de un tipo humano original (el hombre, genérico) a partir del cual otro tipo podría ser mensurado (el femenino, particular). Enyian es (en Yoruba) una palabra neutra, sin género, para todos los humanos (diferente de hembra ([fe]male) o mujer ([wo]man)" (ibidem:33).

2) "Obinrin no es una categoría jerárquicamente ordenada en relación a okunrin (ambas comparten la misma raíz neutra rin)".

3) Ellas son solamente aplicables a adultos. Niños son todos omode. Animales machos y hembras son denominados ako $y$ abo. Plantas son abo cuando producen brotes.

"Asî" - dice ella en la página 33- "en este estudio, los términos básicos okunrin y obinrin son mejor traducidos cuando referidos al macho anatómico y a la hembra anatómica respectivamente pues se refieren apenas a diferencias fisiológicamente marcadas y no tienen connotaciones jerárquicas [...]".

Oyeronke habla también de "ana-sex": ana-machos y ana-hembras (1997:34)

[...] para subrayar el hecho de que en el sentido del mundo Yoruba era posible conocer esas distinciones fisiológicas sin proyectar una jerarquía inherente a las dos categorías sociales. A diferencia de "macho" o "hembra” en el Occidente, las categorías obinrin e okunrin eran exlusivamente categorías relativas a la anatomía y no permitían mayores suposiciones sobre personalidades o psicologías derivadas de esa distinción puramente anatómica. Esto era así, porque no eran elaboradas como una relación de oposición entre sí y, en este sentido, no eran sexualmente dimorfas y 
consecuentemente no recibían atribuición de género. En la antigua Oyo no connotaban jerarquía social ni expresaban masculinidad o femeneidad, ya que esas categorías no existían ni en la vida, ni en el pensamento Yoruba. (ibidem, énfasis mío)

Oyeronke afirma la total ausencia de una estructura simbólica de género en la sociedad Yoruba tradicional (pre-colonial). Para esto, toma como referencia para su argumento una definición standard de género, no como "una propriedad de un individuo o de un cuerpo", sino como "una construcción de dos categorías en relación jerárquica una con la otra [...] embutida en las instituciones", que, como tal, orienta expectativas y ordena todos los procesos sociales (ibidem:39). Y afirma, una vez más, que tal tipo particular de célula ideológica estaría, para ella, ausente en el mundo Yoruba previo a la colonización.

Con respecto a las divinidades del panteón, Oyeronke las sitúa en tres niveles. En el primer nivel está

Oludumare (Dios - el ser supremo), que no tenía identidad de género y es dudoso que ella (o él) fuera percibida de forma antropomórfica antes del advenimiento de la cristiandad y del islamismo en el país Yoruba. (ibidem:140)

Para apoyar esta afirmación, la autora cita fuentes que pretenden demostrar cómo los estudiosos de la época posterior a la cristianización pasaron a representar Oludumare con atributos masculinos y lo llamaron "Él", usando la tercera persona en masculino, sin que nada los autorizara a hacerlo así. Sin embargo, la autora no proporciona evidencias de fuentes que le permitan hablar de la representación previa de ese dios como no antropomórfico o, en su defecto, como antropomórfico, pero sin atributos de género. Sin embargo, un dios supremo exento de antropomorfismo sería una verdadera rareza en la historia de las religiones.

En el segundo nivel del panteón de divinidades, de acuerdo con Oyeronke, están los orixas, sobre los cuales dice: "Aunque eran orixas ana-machos (anatómicamente masculinos) y ana-hembras (anatómicamente femeninos), al igual que en otras instituiciones, tal distinción no tenía consecuencias; por consiguiente, es mejor describirla como una distinción sin diferencia”. Y apoya su aseveración mencionando que algunos orixas de sexo anatómicamente distinto compartían algunas cualidades (la "ira", dice ella, de Xango y Oya, por ejemplo), o que algunos mudaban de sexo entre una localidad y otra. 
De hecho, eso también sucede en el Nuevo Mundo, donde las divinidades tienen personalidades particulares y estas personalidades, a su vez, son subdivididas a partir de un criterio de género, a pesar de las similaridades que porventura puedan presentar. También aquí el género del "santo" puede alterarse con el cambio de localidad o de tiempo. Xango es sincretizado con las imágenes de Santa Bárbara, en Cuba, y São João en Brasil; y de Oya (Iansã) se dice que fue hombre (macho) en el pasado mítico, y pasó a ser mujer (hembra) después de tornarse esposa de Xango (cf. Segato, 1995a). En Bahia, Logunede es mitad del año masculino y la otra mitad, femenino. Pero esto, como mostraré, no me parece ser una evidencia de la inexistencia de una construcción simbólica marcada por el género en esta cultura sino, justamente, un comentario codificado que revela la existencia de una concepción de género particular, un enunciado sobre el género y un discurso político construído en términos de género.

Leyendo a Oyeronke, uno es llevado a preguntarse por qué si, como ella afirma, la anatomía carecía de significado social entre los Yoruba, los orixas, divinidades libres y por encima de las restricciones humanas, eran sin embargo dotados de anatomía y comportamiento sexualizados en sus representaciones mitológicas. Cómo sería posible que tal anatomía ideal de los dioses del panteón, puro significante, nada significase para los asuntos humanos? En otras palabras: por qué los orixas tenían, en su representación mítica, cuerpos marcados por el dimorfismo anatómico y predicados de género si esto no tenía, como afirma Oyeronke, cualquier consecuencia en las relaciones sociales, si esto no expresaba nada relativo a un imaginario de género entre los seres humanos?

En el tercer nivel, la autora sitúa el culto de los ancestrales "[...] machos y hembras, venerados por los miembros de cada linaje y reconocidos anualmente en el ritual de enmascarados del Egungun”.

[...] el oficio sacerdotal dedicado a los varios dioses podía ser ejercido por machos y hembras [...]. La religión Yoruba, así como la vida civil Yoruba, no articuló el género como una categoría [...]. Los roles de orixas, sacerdotes y ancestrales no dependían del género. (ibidem:140)

Para Oyeronke, el idioma dominante en la sociedad Yoruba era el idioma de la senioridad, es decir, del rango relativo a la edad. Lo que efectivamente importaba, e importa, afirma la autora, es si la persona es un niño, un adulto o un viejo: omo significa niño, cría (1997: 40-41); solamente más tarde, a partir del siglo XIX, 
"omokunrin (niño) y omobinrin (niña), hoy términos corrientes, pasaron a indicar ana-sexo para niños" (1997:41). Los términos demuestran que lo que es privilegiado socialmente es la juventud del niño, no su anatomía diferenciada. Por la misma lógica, cuando se llama a alguien de Iya (madre) o baba (padre), de obinrin (mujer) o okunrin (hombre), lo que importa es que ellos son identificados como adultos, en edad de reproducción."El atributo más importante que esas categorías indican no es el género; antes, es la expectativa en que personas de una cierta edad tengan hijos". Iya (madre) o baba (padre) "[...] no son exactamente categorías de paternidad o maternidad [...]. Son igualmente categorías de adultez, en la medida en que también son usadas para referirse a personas mayores en general. Y lo más importante es que no se oponen binariamente ni son construídas una en relación a la otra" (1997: 41). Con esto la autora enfatiza que el predicado de edad relativa y de mayoridad es más relevante, en vocativos como Iya o baba, que las implicaciones de género que estas dos palabras parecen connotar.

Por lo tanto, Oyeronke no tiene dudas de que, en el universo Yoruba, la senioridad prevalece sobre el género, y puede inclusive introducir inversiones en el orden del género si lo observamos desde la perspectiva de género occidental. Por ejemplo, una obinrin mayor o investida con una posición religiosa puede ser considerada padre de un okunrin. Oyeronke recorre a las observaciones de Johnson para describir la relación entre el soberano de Oyo y la obinrin oficial del palacio que comanda el culto a los espíritus de los reyes difuntos:

[...] el rey la considera como su padre y se dirige a ella como tal [...]. Se arrodilla al saludarla y ella retribuye el saludo arodillándose, nunca reclinándose sobre el codo como es costumbre de las mujeres cuando saludan a sus superiores. El rey no se arrodilla ante ningún otro que no sea ella, y se postra delante del dios Xango y de aquellos que se encuentran poseídos por esta divinidad, llamándolos "padre". (Johnson, 1997:37-38)

Como mostraré más adelante, una estructura impresionantemente similar a ésta puede ser encontrada en el culto Xango, en Recife, Brasil; sin embargo, mi interpretación resulta de un modelo diferente. De hecho, en Brasil, también sucede que, dependiendo de su senioridad y del género de su orixa, uma sacerdotisa puede ser ritualmente abordada como un "padre", y una esposa, también dependiendo de su orixa tutelar, puede ser considerada más viril que un marido. Pero todavía más interesante es el hecho de que el saludo ceremonial llamado odobale está desconectado de la anatomía de 
un modo aún más radical que en el mundo Yoruba africano. En el dobale africano, de acuerdo con Oyeronke (1997:36), la reverencia ejecutada depende del sexo de la persona que saluda; en Brasil depende del sexo de su orixa tutelar.

Para Oyeronke:

[...] el desafío que la concepción Yoruba presenta es un mundo social basado en relaciones sociales, no en el cuerpo. Eso demuestra que es posible comprender los roles reproductivos distintos de obinrin y okunrin sin usarlos para crear una jerarquía social. En la lógica cultural Yoruba, la biología es limitada a cuestiones como el embarazo, que tiene que ver directamente con la reproducción [...] A eso llamé una distinción sin diferencia social" $[\ldots]$

Los términos obinrin y okunrin indican meramente las diferencias fisiológicas entre las dos anatomías cuando ellas tienen que ver con procreación e intercurso (sexual) [...] y no indican categorías de género que connotan privilegios y desventajas sociales. Además, no expresan dimorfismo sexual, porque la distinción que indican se refiere exclusivamente a las cuestiones relativas a la reproducción $[. .$.

Un superior es superior independientemente del tipo de cuerpo que presenta. [...] Ori (la cabeza, el principio vital) no tiene género. (1997:33, 34-35, 38).

Tenemos aparentemente aquí un sentido inusual para el término dimorfismo, pues, me pregunto: quiere la autora decir con eso que hay más de dos partes morfológicamente diferenciadas interviniendo en la procreación?

Alternativamente, lo que sostengo es que, independizados del cuerpo, los términos de género permanecen como un idioma para relaciones sociales y organizan algunos aspectos de la interacción social. Por el contrario, para Oyeronke solamente los papeles reproductivos permanecen, colados, vinculados, colapsados, fundidos al cuerpo. Anticipando parte de mi propio argumento, eso me parece muy próximo al papel central que los miembros del culto Nagô o Ketu (Yoruba) en Brasil atribuyen a la anatomía. en la división del trabajo ritual. Es justamente el ritual -y no la cópula o intercurso sexual- lo que reproduce los linajes religiosos africanos en Brasil y, significativamente, la división de los roles rituales por género es el único ámbito de la vida socio-religiosa que obedece el trazado del dimorfismo sexual, orientándose por éste. Es decir, en el ambiente del candomblé, las formas anatómicas sólo son determinantes en su comando de la división del trabajo ritual. Sin embargo, es el as- 
pecto simbólico del dimorfismo, y no su dimensión biológica, lo que realmente cuenta -ya que nos encontramos en el reino de la reproducción religiosa y filosófica de una Africa espiritual, y no biológica ni racial. Solamente cuando lo encaramos a partir de la amplia perspectiva ofrecida por el Nuevo Mundo se torna posible identificar el núcleo de lo que está en juego en esta discusión y también comprender que la tesis de Oyeronke, bajo su aparente radicalidad, vehicula un pensamiento convencional y acrítico.

Si, por un lado, la reproducción y la continuidad del África en Brasil se procesan a través de roles rituales anatómicamente distribuidos, por otro lado eso contrasta con todas las otras esferas -social, psíquica, afectiva y sexual- de la vida religiosa. Para dar un ejemplo, en el ambiente de los cultos afro-brasileños, la reproducción, la crianza de los niños y la organización doméstica son concebidas como separadas de la biología. La unidad familiar -y la doméstica- del culto, la así llamada "familia de santo", operativa en todos los ámbitos de la vida, no está basada en el presupuesto de la sustancia biológica compartida, sino en la iniciación, es decir, en la substancia ritual compartida (llamada "ashé" e inoculada en el cuerpo del iniciado por su "padre de santo"). También las personalidades y la orientación sexual están libres de determinaciones biológicas. Y sería posible proseguir interminablemente dando ejemplos de un ambiente de género que opera con libertad en relación al dato anatómico y en el que las nociones Yoruba descriptas por Oyeronke pueden ser vividamente reconocidas. Sin embargo, un claro esquema de género, lejos de encontrarse ausente, da el vocabulario para categorías importantes de la vida social, aunque subvertiendo el sistema occidental.

Oyeronke incurre, por otro lado, em innumerables contradicciones. Una de ellas, por ejemplo, cuando, mientras niega cualquier connotación de género en las palabras oko y aya, traza la equivalencia de esos términos con posiciones en el ambiente familiar. Dice ella: "[...] la traducción de aya como 'esposa' y oko como 'marido' impone construcciones sexuales y de género que no hacen parte de la concepción Yoruba” (1997:44). Y aduce: "[...] oko y aya [son, respectivamente] dueño/de dentro y no-dueño/de fuera [haciendo referencia a la virilocalidad vigente en la cultura] en relación al ilé como espacio físico y símbolo del linaje. Tal posición relativa de dentro/de fuera era jerarquizada [énfasis mío], con el de dentro siendo el término sénior, privilegiado", Entonces, clara e innegablemente, los términos de género están aquí asociados con el status. En una casa de familia, dice Oyeronke, todos los miem- 
bros más antiguos de la casa, machos y hembras, eran considerados oko -maridos, séniores- para la nueva esposa que llegaba-aya-, aunque ella supuestamente tenía una relación marital sólo con su cónyugue; cuando éste último moría solamente los 'ana-machos' (anamales) más jóvenes que él en la casa podían reclamar derechos sexuales sobre ella, "en la medida en que éste es un mundo heterosexual' (sic, énfasis mio), y cuando un oko ana-hembra (anafemale sénior) reclamaba derechos de herencia sobre la viuda, el acceso sexual pasaba para uno de sus hijos ana-machos (anamales).

Significativamente, Oyeronke nos hace saber también que, mientras hombres y mujeres podían ser oko para otros hombres y mujeres en función de su senioridad, ana-machos (anamales) no podían ser aya (esposas) de ningún ana-macho (anamale) o ana-hembra (anafemale). Ellos sólo podían ser aya para los orixas a los cuales prestaban culto y recibían en posesión, lo que quiere decir que los machos anatómicos no cruzaban la frontera decreciente de género en el campo social. Definitivamente, entre los Yoruba pre-coloniales, los machos anatómicos estaban conectados a una condición de status y prestigio que no combinaba con el papel social propio de esposa, excepto bajo el comando de las divinidades. Nos sorprende que un punto de tal importancia, con todas sus consecuencias, pase completamente inadvertido por la autora. Lo que, creo, termina por colocar una seria limitación a la eficacia de su modelo.

No obstante, a pesar de las dificultades etnográficas que su modelo no consigue superar o, quizás, precisamente por no conseguir superarlas, la autora nos ofrece un vislumbre de las complejidades del género entre los Yoruba, comunicando con su descripción el alto grado de maleabilidad del sistema. Esta maleabilidad del género en el universo Iorubá tuvo sin dudas un papel crucial en la relocalización de la cosmología propia de esta cultura -y de las prácticas a ella asociadas- en el Nuevo Mundo, particularmente en Brasil y, más tarde, en la reciente onda de expansión para el sur, en los nuevos territorios de Argentina y de Uruguay.

\section{Lorand Matory}

Lorand Matory publicó Sex and the Empire that is no more en 1994, cuando era profesor asistente de Antropología y Estudios Afro-Americanos en la Universidad de Harvard. Su texto también ofrece un testimonio de la existencia de una compleja construc- 
ción de género en el mundo Oyo (Yoruba) tradicional. Él también hará un gran esfuerzo en su tentativa de representar etnográficamente esa complejidad -que lleva los esquemas de género de la cosmología Yoruba y las prácticas religiosas asociadas a una condición de casi inefabilidad-por medio de la formulación de un modelo basado en la idea de "transvestimiento". Sin embargo, Matory afirma que "las mujeres preservan la imagen paradigmática de la mujer casada ("married wifeliness") no apenas en las religiones de los orixas, sino a través de todo el espectro religioso Yoruba" (Matory, 1994:108).

En el modelo de Matory, lo que él denomina "iconografía sartorial", " un ritual diacrítico (idiosincrático) de género y gestos asociados al trabajo marcan lo que es propio de las mujeres -la ropa que usan o tallas/esculturas/figuras de mujeres "que se arrodillan para ofrecer servicio y sacrifício, cargar fardos en la cabeza y/o amarrar un bebé a la espalda" (1994:108). Pero, a pesar de su estricta dependencia de estas marcas emblemáticas -y no anatómicas- el vínculo con las determinaciones biológicas es, sin embargo, establecido a través de las mujeres: "el status marital y reproductivo de las mujeres afecta directamente su posición en todas las organizaciones religiosas locales. La menstruación compromete la participación de mujeres en edad de concebir" (1994:107-108).

El transvestitismo (transvestitism) es, para Matory, el principal idioma "irónico" de las estructuras de género em la sociedad Yoruba. Éste permite, por ejemplo, que personas del mismo sexo entren en una relación social como oko y obinrin (con o sin implicaciones sexuales). Sin embargo, la posición paradigmática del cuerpo de la mujer y sus atributos anatómicos, gestuales o sartoriales como significantes de una posición femenina relacional (aunque un cuerpo de hombre pueda también ocupar esa posición), revela la existencia de un mapa cognitivo construído claramente en términos de género.

Para Matory, ese mapa no es verbal ni regido por categorías léxicas; es preferencialmente visual e inscripto por íconos, gestos y marcas visuales. Ya Oyeronke niega la importancia de la visualidad entre los Yoruba y afirma la dominanción de lo auditivo. Ella también afirma, como vimos, que no existen palabras en la lengua Yoruba para masculino y femenino em cuanto posiciones o personalidades opuestas (Oyeronke, 1997:34), siendo apenas encontradas aquellas que indican las posiciones relativas de marido y esposa (oko e obinrin). En el modelo de Oyeronke, permanecemos interrogándonos por la razón de ser de categorías verbales, estatuaria y cos- 
tumbres definidas en términos de género, si ninguna de ellas está destinada a tener cualquier significación en la vida social. En el modelo de Matory, cabe preguntar el por qué de las prácticas generalizadas de transvestitismo si las jerarquías sociales de género permanecen en su lugar, intocables, en la ideología de género Yoruba.

Ambos autores parecen estar de acuerdo en cuanto a la existencia de un modelo en el cual el género sigue un esquema radicalmente diferente del occidental. Pero, mientras en el texto de Oyeronke encontramos beligerancia y la premisa de la colisión de civilizaciones mutuamente intraducibles, una de ellas derrotada y colonizada, que permanece solamente como um anti-paradigma civilizatorio sofisticado y puro, en Matory hay una lección para llevar a casa, como trataré de demostrar.

Creo yo que el discurso que acabé de mostrar, el de Oyeronke, carga su propio hibridismo, inserto en su enunciado por su interlocutor preferencial: el Occidente. Al afirmar la existencia de una África antigua libre de jerarquías de género -y ella es clara sobre eso- defiende la idea de una Africa pre-colonial en estado puro, y retrata la introducción de relaciones sociales marcadas por la jerarquía de género como indicador de una África occidentalizada. El texto de Matory, al que pasaré a referirme, tiene también un interlocutor en sombras. Mientras Oyeronke es una antagonista, Matory es un reformista conservador. Pues me parece que lo que hace es traer la idea del transvestitismo y de la centralidad de género para la polis, para sus conciudadanos, para su país. En su etnografía -y es lo que intentaré demostrar-, él habla en nombre de una estructura de género jerárquica que puede permanecer como un principio organizacional de una sociedad tambiém jerárquica, a pesar de que las personas cambien de piel y se transvistan. La que propone es una célula de relaciones de género estable, que no amenaza o subvierte el sistema, aunque pueda introducir transformaciones.

Los etnógrafos, como los intelectuales en general, jamás dejan de estar politicamente orientados, guiados por intereses y valores, vehiculando mensajes crípticos para sus pares, trayendo argumentos para casa.

Matory nos presenta un mundo donde el transvestitismo conduce siempre a una organización asimétrica, constituída en términos de género, jerárquica: "esposas machos y maridos hembras son actores centrales en el reino y en la aldea Oyo-Yoruba", dice él (1994:XII); "[...] todas las mujeres son maridos para alguien y simultáneamente esposas de múltiples otros” (ibidem:2); el 
transvestimiento masculino (de los machos) en Oyo-Yoruba no es sólo un idioma de dominación ni apenas la evidencia de la independencia de las categorías de género con respecto al sexo biológico, pero una práctica que "transforma las categorías de género existentes" (ibidem:3). El hecho es que, en el modelo de Matory, la práctica de transvestitismo reduce las categorías de género al sentido estrecho de universalizar sus estructuras jerárquicas en el campo social, proyectándolas, además, mucho más allá del campo de los roles de género y de la sexualidad. Y es en ese sentido que Matory toma de Marilyn Strathern la idea, formulada para las sociedades del Pacífico, que "[...] la inequidad sexual/de género es el 'idioma' irreductible por medio del cual hasta la ineqüidad existente entre personas del mismo sexo y género es expresada y comprendida" (ibidem:176-7).

En el centro del modelo de Matory encontramos la imagen -común para describir la experiencia del trance en las religiones africanas del Viejo Continente y en la diáspora del Nuevo Mundo- de seres humanos en estado de posesión "montados" por dioses. Ciertamente es un modelo explicativo demasiado simple para un sistema de pensamiento tan complejo como el que pretende ecuacionar. Matory nos dice:

[...] el vocabulario y el código para el vestuario de las religiones de posesión [...] iluminan la estructura de aquella relación. Recién iniciados de Yemoja, Osun, Obatala y Xango [...] son conocidos específicamente como "novias de la divinidad". Ellos usan ropas o atributos de mujer. Y se dice que el dios monta a aquéllos en quienes baja en posesión (ibidem:7).

El dios es invocado como "marido" y "señor" por los devotos. "El concepto de 'montar' (gigung) asemeja al sacerdote (elegun) a un corcel real (esin) y a una esposa real (ayaba)" (ibidem:135). Potes y calabazas son también íconos del ordenamiento jerárquico propio del casamiento patrilineal.

La jerarquía de género no representa la superioridad de un sexo biológico sobre el otro y sí la asimetría, tal como ésta se expresa por la relación de los géneros en el casamiento.

Con el casamiento, una mujer se torna una esposa (iyawo) no apenas para el hombre que ella desposa (okogidi), sino para todos los agnates, machos y hembras, emparentados a través del casamiento con ese hombre, y para las mujeres que estaban casadas con él y con sus agnates con anterioridad a su llegada a la casa. Recíprocamente, no apenas el hombre que ella desposó, pero todos sus agnates son clasificados como sus maridos (oko). (ibidem:105). 
Vemos, aquí, una descripción idéntica a la ofrecida por Oyeronke para las relaciones de la esposa que llega a su nueva casa después del casamiento. O sea, esposa y novia, en este idioma social basado en el lenguaje de género, significan júnior, subordinado. Esa organización típica es notada también por Oyeronke, como vimos, pero mientras ella la interpreta como ausencia de género, Matory la entiende como la generalización de los términos de género como sistema de clasificación en un campo social jerárquico.

Género y sexualidad se tornan el idioma característico de las transiciones jerárquicas de la sociedad Yoruba tradicional. Tal vez, la más clara expresión de ese arreglo sea proporcionada por el hecho de que, cuando el sacerdote sénior (elegun) recibe su orixa en posesión, lo hace en calidad de "novia" del dios, y es "montado" por el dios como una metáfora sexual. Y muchos creen que quienes reciben Xango, "montados" de esta forma por el dios en posesión puede, a su vez, ellos mismos, "montar", es decir, realizar el acto sexual con una mujer de la audiencia (ibidem:170). Se revela aquí el hecho de que el mismo actor social actúa simultáneamente como subordinado en una relación y como dominador en la otra, femenino en una pareja, masculino en la otra. Este lenguaje de la circulación del posicionamiento de género yo lo señalé también para Brasil, con base en mis materiales etnográficos. La idea es la misma. Si embargo, en Brasil, y según mi interpretación, su impacto y finalidad no se orientan a organizar las relaciones sociales y representar las jerarquias politicas y sociales mediante el uso del idioma de género, sino, justamente a la inversa, a desestabilizar el patriarcado, reírse de él, ironizarlo, no apenas en un sentido discursivo pero también en un sentido literal, efectivo. En mi análisis del material brasileño, la aplicación de los términos de género induce a la subversión de su uso habitual. Las posibilidades del sistema de género que Matory llama de "irónico" para los Yoruba son llevadas a una radicalidad extrema en Brasil. Veamos algunos ejemplos.

Me baso aquí en mis materiales de la tradición Nagô (Yoruba) de Recife, cuya estructura de género, a pesar de diferencias mitológicas circunstanciales, permanece reconocible en el Candomblé en general. El panteón de culto presenta a primera vista la apariencia de un grupo familiar formalmente organizado. Pero, tan luego se atraviesa la superficie encontramos un padre-Orixanla (Obatalá en Cuba), un patriarca, quien, a pesar de su personalidad en última instancia vengativa, no ejerce su prerrogativa de autoridad sobre los otros orixas, ya que es lento y débil. Una madre-Yemanja-quien, no obstante su apariencia suave y pulida, es falsa y traicionera "como 
el mar" (dicen: "del mar, se ve apenas la superficie y nunca las profundidades" -ecos de una memoria codificada del papel traicionero del mar en el middle pasage pueden ser oídos aquí; una madre que no crió a sus propios hijos, haciendo con que fuesen cuidados por una madre de leche-Osum. Una diosa de la fertilidad, Osum -que no es la que gesta, sino la que cuida-, haciendo eco, aquí, de forma codificada, una vez más, la memoria de gran duración histórica en el Brasil, de la separación entre la madre blanca, señora de la Casa Grande, y la nodriza esclava. Un padre -Orixanala- que cría muy amorosamente una hija, su preferida, que sin embargo es fruto de su mujer-Yemanja- en un caso de infidelidad con un dios más poderoso -Orunmilá. Iemoja. Una esposa -Iansan (Oya) - de quien se dice que es más "viril" que su marido -Xango-, porque ella comanda los espíritus de los Muertos - eguns-, quienes dejan a Xango aterrorizado. Un primogénito de la dinastía muy trabajador y aplicado -Ogum-, cuyo trono es usurpado por el astuto y consentido hermano menor-Xango- con la connivencia de la madre-Yemanja-, que en el momento percibe la maniobra pero no hace nada por evitarla pues le teme más al desorden que a la injusticia. Un padre-Orixanla-, contempla omiso e impotente la injusticia que se comete en el acto de la coronación. Un reino en el que la madre, y no el padre, tiene la prerrogativa de coronar al rey y controla oficialmente los asuntos del reino.

Por lo menos dos episodios de seducción homosexual entre las divinidades son narrados -esto sin mencionar aquí las prácticas sexuales y el modo de vida que se apoya en esta mitología. En fin, invocada y aludida en la conversación ordinaria, una innumerable serie de inversiones transforma esa mitología aparentemente convencional y jerárquica en un discurso irónico sobre la sociedad brasileña, donde no apenas la determinación biológica es removida de su lugar usual de soporte de la ideología, sino también el patriarcado y la jerarquía son desestabilizados por las prácticas diarias. Las fundaciones patriarcales de un estado "doméstico" privatizado son también cuestionadas. Una duda fundamental sobre las estructuras de género en que se asienta la moral social dominante es inoculada en el sistema político en su totalidad (cf. Segato, 1995a y 1995b).

\section{Géreno y sociedad: del mundo Yoruba al Occidente}

Muy por el contrario, de acuerdo con Matory, en la región Yoruba el sistema de género y su vocabulario son usados para crear 
un régimen social marcado por posiciones relativas rígidamente jerarquizadas que atraviesan el ámbito entero de las relaciones humanas y de los humanos con las divinidades. Infelizmente, su obra no responde a la pregunta central: ¿cómo eso afecta efectivamente el género y el ejercicio de la sexualidad?. La gramática de género es abordada meramente como el idioma de las jerarquias sociales. El argumento enfatiza que los iconos del género se destinan fundamentalmente a sustentar y expresar la jerarquía social. A pesar de lo que el autor afirma, nos encontramos frente a un sistema que, aunque independiza el género del sexo biológico, no rompe y ni siquiera corroe el régimen de género, entronizándolo como paradigma de todas las relaciones en un mundo intensamente moldeado por las jerarquías.

En el sistema Yoruba, afirma el autor que comento, el género existe a través de predicados, y estos predicados son sociológicos, relacionales. El femenino, como Matory nos lleva a comprender para el mundo que describe, es un atributo relativo a la posición de la novia, y el masculino no es un predicado del hombre sino del marido en su posición marital. El género, una vez más, es posición en relación y no esencia biológica (Matory, 1994:164). Pero, al final, una matriz rígida de poder y prestigio jerárquica y heterosexual persiste en este orden de género "técnico" - para citar el término que Matory utiliza-, altamente artificial y auto-consciente. La distribución de derechos y deberes y el código de etiqueta son determinados por el "género", entendido de esta forma: como el lenguaje de los rangos relativos. Posiciones relativas móviles son contenidas dentro de un paradigma institucional fijo.

Más que afirmar o desarticular las categorías de género existentes, este transvestitismo sagrado sustenta transformaciones de género que lo tornan el más denso de todos los emblemas locales de poder (ase) y de subjetividad. (ibidem:175)

Aquí, el acto de "montar" arma la escena de la asimetría (sexualmente, ritualmente y, en tanto alegoría, socialmente). "El género, entonces, es el idioma de las relaciones entre dioses y sacerdotes, caballeros y caballos, padres e hijos, viejos y jóvenes, reyes y plebeyos" (ibidem:177). Así, "como una fuente de predicaciones metafóricas sobre jerarquía política, privilegio económico y salud personal, el género deja de ser género como nosotros lo conocemos" (ibidem:177-178). Y el autor se pregunta: "El cross-dressing afirma o corroe los roles hegemónicos de género? Cuáles son las fronteras que el cross-dressing ultrapasa?" (ibidem:202).

Yo diría que él mismo proporciona las evidencias para la respuesta: ninguna frontera es cruzada. Las posiciones fijas de hom- 
$\mathrm{bre/mujer} \mathrm{son} \mathrm{sustituidas} \mathrm{por} \mathrm{las} \mathrm{posiciones} \mathrm{móviles,} \mathrm{relacionadas,}$ marido/esposa en el centro del sistema. La estructura marido/esposa atraviesa el sistema organizándolo jerárquicamente. Maridolesposa se torna la metáfora permanente de la polis religiosa organizada jerárquicamente. Así, "el transvestitismo sartorial y cefálico" practicado en Oyo Yoruba no es visto por Matory como "una inversión ritual con la intención de manifestar el poder del desorden [...]. Ni surge para minar las desigualdades del poder relativo al género" (ibidem:211). Antes, y claramente, Matory habla de la conservación de un orden por medio de la simbolización secundaria de ese orden $y$, así, el transvestitismo es conducido al centro del orden institucional, como una fuerza conservadora en la estructura. Eso, en el sentido preciso de que, en las propias palabras de Matory,

[...] el transvestitismo no es un fenómeno marginal. Es central, una vez que se encuentra codificado y diseminando en un estado imperial y hoy responde a las aspiraciones más profundas de centenas de millares de nigerianos, beninenses y, como podemos ver, brasileños. (ibidem:215)

Mientras Oyeronke dice al Occidente que el género no existía entre los Yoruba pre-coloniales y reivindica, con esto, la diferencia de su propio mundo y su propia diferencia, el recado de $\mathrm{Ma}-$ tory es que el transvestitismo y la transposición de esquemas fijos de género a anatomías variables es un idioma eficaz de organización social, tan o más eficiente que una concepción de género asentada en la biología. Sobre todo porque, como el modelo de Matory sugiere, lo que cuenta es la lógica (jerárquica) de la matriz de género, no la anatomía que lo incorpora.

\section{El componente de género en la difusión de la visión del mundo yoruba}

El complejo sistema de género que los autores citados, de una u otra forma, intentan describir y que operaba en la polis religiosa de los Yoruba tradicionales parece haber sido unos de los pilares de la sólida expansión de la religión y de la cosmología Yoruba en Brasil, y de éste para otros países en la actualidad. Efectivamente, cualquiera que haya sido o todavía sea en el África la organización precisa del sistema al que los autores que cité apuntan, es evidente que un extraordinario trabajo de preservación tuvo lugar en el Nuevo Mundo. No fue meramente preservada la idea elemental de que el santo "monta" a su medium, caballo, o tutelado, tan difundida en el mundo afro-americano para hablar de la posesión, 
sino también, y muy especialmente, las complejidades y abstracciones del manejo social de género. Este trabajo de preservación no se restringió exclusivamente a los aspectos formales, ritualísticos y litúrgicos de la tradición Yoruba en el Nuevo Mundo, que inequívocamente se vinculan al modo de sociabilidad indicada por los autores citados para el África, sino que se amplió y radicalizó afectando los aspectos de la construcción de género más inefables y esquivos para las categorías sociológicas como la sexualidad misma.

En mis escritos utilizo la expresión "codex afro-brasileño" para hacer referencia a este núcleo duro y permanente de corte antiesencialista que atraviesa las prácticas y los saberes de la cultura del Candomblé (ver Segato, 1988). Con el término "codex" intento enfatizar la redundancia y la repetición significativa de algunos motivos. Estos motivos repetidos apuntan para la existencia de un código fijo en acción por detrás de las prácticas observables que, a su vez, revelan la existencia de este nivel más profundo, oculto, de una inscripción críptica que se manifiesta, sin embargo, todo el tiempo en el discurso -mitológico, social y ritual- de las religiones de origen Yoruba en el Nuevo Mundo y, especialmente, en el Brasil.

Para mí fue, debo decirlo, particularmente conmovedor el encuentro con los dos libros referidos, publicados ambos algunos años después de mi primer ensayo sobre el tema. Otros académicos también pusieron atención en las peculiaridades de los sistemas de género religiosos Africanos-Americanos y la presencia de los homosexuales en ellos en Brasil (Landes, 1940, 1967; Ribeiro, 1969; Fry, 1977, 1986; Wafer, 1991; Birman, 1995) como en la Santería de Cuba (Dianteill, 2000) y Vudú Haitiano (Lescot, 2002). No obstante, la especificidad de mi abordaje es justamente que afirmo que el sistema de género es el factor estructurante que hizo posible la continuidad de esta tradición, constituyendo el núcleo y no un elemento superfluo o adicional, que podría estar ausente sin afectar la cultura, la cosmovisión y la sociedad afro-americana del candomblé como un todo. En otras palabras, trato a los comportamientos homosexuales, tantas veces señalados como una constante en la sociabilidad y la sexualidad de los cultos, no como un elemento separado, sino como consecuencia de una construcción particular del sistema de género que, a su vez, no es meramente un dato más sino que constituye la estructura central y fundamental para comprender la filosofía, la cultura y la religión en cuestión.

Mucho fue dicho sobre las razones por las cuales la civilización Yoruba dominó otras culturas africanas que vinieron para el 
Nuevo Mundo. Dos aspectos son en general apuntados como cruciales: 1) la llegada masiva de um contingente Yoruba después del cierre oficial del tráfico intercontinental de esclavos; y 2) las sólidas instituciones de poder del imperio Yoruba en Africa. De lo que pude observar en los ambientes religiosos de origen africano en Brasil y, más tarde, durante los últimos veinte años, sobre la reciente expansión de las religiones hacia el sur para instalarse en los países de la cuenca del Río de la Plata (ver Segato, 1991, 1996), pienso que es posible adicionar un tercer y fundamental factor a esas razones, esto es, la maleabilidad del sistema de género y con él la flexibilidad y el anti-esencialismo de los arreglos familiares.

Para la primera ola, yo diría -brevemente, ya que he escrito extensamente sobre el asunto- que el uso no esencialista de los términos de género y de familia encontraron un terreno fértil en el ambiente colonial brasileño. Eso fue así porque las parejas constituídas y sus descendientes no pudieron estabilizarse como un grupo familiar en el Brasil, donde las familias fueron dispersadas; la proporción demográfica entre hombres y mujeres en el contingente de esclavos fue extremamente inadecuada; y el casamiento entre esclavos fue activamente desestimulado y obstaculizado por un largo período y en casi todas las regiones del país (Segato, 1996). En consecuencia, una construcción de género y una terminología para la organización familiar libres de la determinación biológica y no fijados o coaccionados por significantes anatómicos se adecuaba idealmente a la situación. Aún más, en ese nuevo ambiente, el sistema entero pasó a afectar también la sexualidad, y no operó meramente como un idioma para el status social, como el descripto por Matory.

En la segunda ola, las religiones afro-brasileñas de base Yoruba (como el "Batuque" de Puerto Alegre y el Candomblé de Bahía) se expandieron para Argentina y para Uruguay. En el interior de esas sociedades nacionales, según mi interpretación (Segato, 1991, 1996), ellas propiciaron la demarcación de un espacio de diferencia y de representación simbólica para grupos carentes de libre expresión o visibilidad y, entre esas minorías, la minoría homosexual, tradicionalmente asfixiada en los países hispánicos y con escaso espacio para la auto-representación y el reconocimiento, encontró su nicho de expresión.

El mundo Yoruba fue restaurado en Brasil en torno del culto de los orixas como encarnaciones modelares de tipos de personalidad. La personalidad fue la noción que permaneció cuando las ataduras regionales y de linaje que sustentaban los cultos de orixas 
particulares y los vinculaban a la familia y a la localidad se perdieron como consecuencia de la trata de esclavos. El casamiento y la célula paradigmática oko/obin descripta por Matory también se perdió, y la línea de descendencia fue transferida para una familia no-biológica ritualmente constituída y reproducida. La genealogía fluye a través de la "madre" o del "padre de santo" para los "hijos de santo", esto es, por iniciación como miembros del culto. Iyawo, por ejemplo, que significa "esposa" o, inclusive, "esposa de orixa" cuando referida a un sacerdote del país Yoruba, pasó a ser comprendida, en la transcripción brasileña, como "hijo" o "hija" de santo, y el ritual de "salida de Iyawo" alude a la presentación ceremonial del nuevo iniciado en la sociedad del culto. Los orixas permanecieron subdivididos por género, lo que reforzó su funcionamiento, más nítidamente que nunca, como clasificadores de las personalidades de sus tutelados en masculinas y femeninas. En este verdadero zodíaco de género, una persona con cuerpo femenino puede tener una personalidad clasificada como masculina si su divinidad tutelar es masculina. En ese caso se dirá que su "santo dueño de la cabeza es un santo -u orixa- hombre". Y una persona con cuerpo masculino puede ser, de la misma forma "hija" de una orixa "mujer". En este modelo, es la personalidad lo que se encuentra predicado por el género, y la anatomía ideal, paradigmática de los orixas, opera como el significante de esa diferencia. Al mismo tiempo, la androginidad y la transitividad de género también se encuentran presentes en el sistema, incorporadas en la mitología de algunos orixas: Logunede, en Bahía, es seis meses del año masculino y seis meses, femenino, y Oya habría sido masculina en el pasado, tornándose femenina en tiempos más recientes, después de su casamiento con Xango, aunque exhibe todavía una personalidad viril. Un continuum es trazado a lo largo del precurso de los orixas en su calidad de personalidades, lo que resulta en que algunos santos femeninos sean considerados más viriles que otros y que hayan grados de masculinidad para los "santos-hombre", al punto que, según la óptica de algún trazo específico del carácter, un santo femenino puede ser entendido como "más viril" o "más masculino" que un santo masculino en el desempeño de una acción o tarea particular.

La profesión sacerdotal es el ámbito que presenta la organización más próxima a la descripta por Oyeronke. Aunque contemple roles rituales de género que nítidamente siguen la división anatómica, eso no implica cualquier diferenciación o especificidad en el desempeño de los roles sociales. La organización Yoruba en el 
Brasil también presenta semejanzas con la descripción de ambos, Oyeronke y Matory, respecto de la casa o unidad doméstica, el agbo ile, llamado ile o "terreiro" en Brasil. Esta casa de culto, donde una "familia de santo" reside y ejecuta sus rituales, es la unidad socio-política de la religión, así como en África el agbo-ile es la célula socio-política de la sociedad. Pero aquí, como dije, la familia se forma sobre la base de vínculos rituales consagrados por la iniciación y la renovación periódica de este voto. De esta forma, el crterio biológico de descendencia queda desplazado a un segundo lugar. En esta organización, el sacerdote -o la sacerdotisa- es el único lider de la unidad doméstica, pero, a pesar de tener su nombre marcado por la diferenciación de género - "padre"o "madre de santo"-, sus derechos y deberes en el ejercicio religioso y social del sacerdocio (no así en el ritual) no presentan especificidades de género; esto quiere decir que el rol social de "padres" y "madres de santo" es exactamente el mismo y es, por lo tanto, un rol andrógino, que no responde a la división de género. De la misma forma, un "hijo" y una "hija de santo", un "hermano" y una "hermana" no poseen obligaciones o privilegios sociales específicos que obedezcan a la división de género.

La labor reproductiva, entonces, corre por cuenta del ritual, que equivale a la cópula sexual, en el sentido que allí, sí, se hace necesaria la contraprestación de tareas rituales específicas de una madre y un padre de santo. Es el imprescindible concurso ritual de ambos, con sus papeles rígidamente distribuídos por género, que permite la reproducción del linaje religioso. Es aquí que veo la mayor semejanza con la aludida "ausencia de género" que Oyeronke atribuye a la tradición precolonial de los Yoruba y la relevancia atribuída por ella exclusivamente al papel reproductivo del dimorfismo sexual.

La senioridad de edad biológica, tal como es descripta para el África por los autores que cito, se transforma en Brasil en un criterio no biológico de senioridad como miembro del culto, es decir, edad como iniciado. Finalmente, el anti-esencialismo y la androginía presentes en el sistema como un todo tienen impacto en las prácticas sexuales también, ya que liberan la sexualidad de la ideología de la coacción anatómica, vigente en el país Yoruba de acuerdo a los dos autores citados. Es en este sentido que el sistema brasileño me parece más radical en la disolución de la matriz ideológica heterosexual y jerárquica, y más distanciado de la estructura simbólica occidental.

A partir de lo dicho, es posible reconocer un esquema de cuatro estratos en el sistema de género Yoruba de Brasil: roles rituales 
-anatómicamente marcados-, roles sociales -andróginos-, personalidad -dimorfismo psíquico-y orientación sexual -nómadesiguen reglas independientes de adscripción y no se encuentran atados entre sí por una camisa de fuerza que los vincula y los obliga a una correspondencia rígida con el dato anatómico, como en la ideología dominante del sistema occidental. La interacción entre ellos propicia la movilidad de género y abre sendas para la androginia. El género total de una persona, esto es, de un "hijo de orixa", es consecuencia de una situación siempre transicional en la compleja intersección de estos cuatro estratos. El motivo de la circulación de género se encuentra reiteradamente inscripto en el codex.

Pero es importante notar que, en el mundo del candomblé, a diferencia de lo señalado por Oyeronke, tanto los términos de género como los que denomina posiciones en la organización de una familia nuclear, aún encontrándose dislocados del determinismo biológico y de la coacción anatómica, tienen plena vigencia. Esta vigencia se refiere no a su función habitual de organizar el mundo de la forma descripta por Matory, es decir, como un idioma para denominar, reforzar y reproducir los rangos sociales relativos, sino como un esquema permanentemente subvertido por las prácticas, desgastado por el comentario mitológico, y utilizado de forma tal que las jerarquías que contiene acaban desarticuladas. El uso de términos de género y de la nomenclatura familiar -tanto en el panteón como en la "familia de santo"-constituye un reconocimiento que acata formalmente el paisaje patriarcal vigente y hegemónico en la sociedad circundante, pero que lo devuelve minado y transgredido por el uso. Estamos frente al caso del subalterno que replica el lenguaje del dominador pero, en su utilización, lo trastoca, lo desgasta, lo socava y desestabiliza, erosionando así la dialéctica misma dominador-dominado, blanco-negro, cristiano afro-brasileño.

Se trata de lo que ya describí en otra ocasión como el característico double voicing, la duplicidad de la voz de los afro-brasileños, utilizando la idea Bakhtiniana del carácter responsivo y dialógico de los enunciados. Aludo aquí al hecho que, aun en la repetición del discurso hegemónico y totalizador del dominador, el subalterno -en este caso el afro-brasileño-, al introducir la marca de su posición diferenciada en la nomenclatura utilizada, lo hace duplicando su voz. En un mismo enunciado, manifiesta que reconoce y se curva frente a la presencia del mundo circundante, totalizado y hegemonizado por la moral dominante, pero una escucha sensible y atenta revela que ese enunciado esconde y vela una voz que, aún 
acatando ese léxico, introduce su corrosiva marca de duda, de "malapráctica”, de insubordinación. En ese sentido, el habla del negro se encuentra dividida, es siempre split voice, doble voz, afirmación y negación, ironía. (Segato, 1996; Bakhtin, 1981). En la primera voz, la imagen del mundo se imprime en él como una impronta positiva; en el velamiento de la segunda voz, vemos en negativo la imagen del mundo que lo cerca-le transmite, en ella, a sus pares, en idioma íntimo, un mensaje de complicidad.

El sujeto del que hablo, entonces, no se ve como un otro substantivo; no es ni un sujeto afirmativo de su realidad "otra", "distinta", ni responde de forma reactiva al orden dominante (como la reacción en última instancia inversamente obediente de los fundamentalismos), sino que mimetiza y tergiversa la familia patriarcal con un uso irónico de la misma. Realiza, con esto, lo que he llamado, en otro contexto, una "mímesis progresiva" (Segato, 2001): imita al amo, responde a sus expectativas, toma conocimiento de la imagen que el orden dominante le atribuye, pero introduciendo un elemento de parodia que transforma la obediencia en desacato. Lo que dice es: ese es el mundo, reconozco su existencia y el hecho que debo convivir con él, conozco también mi lugar y mi imagen en ese orden, pero al replicarlo solamente expreso que tomo conocimiento, y no que acato. Se trata de una imitación que detona aquello que imita, como cuando un negro le llama al otro "negro": diciendo con esto que "sabe", y que se burla de lo que sabe.

Es posiblemente Homi Bhabha quien ha producido un marco conceptual más preciso para referirnos a este conjunto de fenómenos, a los que él se refiere usando la idea de hibridismo. El "hibridismo" en Bhabha es un proceso dinámico, pleno de desestabilización, del tipo que acabo de describir, y no quieto, mecánico como lo conocíamos hasta hoy a través de los textos que se referían a los procesos de folklorización y al sincretismo. No se trata simplemente del encuentro de mundos y el bricolage de una nueva realidad cultural por mezcla. Esta nueva noción de hibridismo tiene el valor de colocar al sujeto híbrido en movimiento, lo muestra en su descontentamiento, en su incomodidad dentro de los significantes que, sin embargo, es obligado a utilizar. Se trata de un sujeto que ejecuta el mandato que sobre él pesa, pero lo ejecuta con un resto, le introduce una torción, un matiz, que es, en el fondo,una marca velada de desacato y el rastro de su insatisfacción.

En su texto "Remembering Fanon", con el que introduce la edición inglesa de 1986 de Piel Negra, Máscara Blanca y reinscribe 
Fanon en el pensamiento crítico contemporáneo después de una década de olvido, Bhabha formula una noción de identidad producida como un enunciado adecuado a su destinatario, es decir, como signo para otro. Esta noción de identidad, inspirada en Lacan (1977) ("la transformación producida en un sujeto cuando este asume una imagen"), se encuentra próxima a la idea Bakhtiniana mencionada más arriba de la responsividad del habla, en el sentido de que la voz del interlocutor se encuentra también audible en el enunciado del sujeto produciendo el célebre efecto dialógico o polifónico señalado por Bakhtin. En esta acepción, el hibridismo resultaría de la ambivalencia inoculada en el discurso subalterno por la presencia en él de las expectativas del discurso dominante y su esfuerzo por, siendo otro, adaptarse a estas expectativas. En esta versión del concepto, su epicentro es la ambivalencia del sujeto en el acto de identificarse y significarse - para el otro dominador.

Otra acepción complementaria es la que enfatiza, en el fenómeno del hibridismo, la introducción en el discurso hegemónico de un índice de su diferencia por parte del sujeto subalterno. Para este concepto, Bhabha también se apoya en su lectura del psicoanálisis:

Hay una diferencia importante entre fetichismo e hibridez. El fetiche reacciona [...] fijando el falo a un objeto con anterioridad a la percepción de la diferencia, un objeto que puede metafóricamente substituírlo y, al mismo tiempo, registrar la diferencia. En tanto y en cuanto el se preste al ritual fetichista, el objeto puede tener cualquier aspecto (o ninguno!). El objeto híbrido, por el otro lado, retiene el semblante del simbolo de autoridad pero reevalúa su presencia ofreciéndole resistencia como significante de un Entstellung [dislocamiento, distorcionamiento, tergiversación, desfiguramiento] - después de la intervención [y el reconocmiento] de la diferencia. (Bhabha, 1994a:115. Traducción, énfasis y aclaraciones entre corchetes míos)

En otras palabras, el discurso híbrido retiene el significante del poder pero le retira su valor, aún reconociéndolo ("después de la intervención [y el reconocmiento] de la diferencia"). En esta acepción, el énfasis está colocado en la insatisfacción interna del sujeto respecto al signo que el discurso dominante le lanza, le pone a disposición, para su identificación. El epicentro no está colocado en el otro como interlocutor a quien debo presentarle una imagen y dirigirle un enunciado de mi identidad, sino en el otro como interpelador poderoso, y en el acatamiento parcial y ambivalente de sus 
categorías, incluyendo la que le ofrece como signo de identificación.

Así, cuando el discurso afro-brasileño introduce en su organización social y en su mitología la apariencia de un respeto a la matriz de género y a la familia patriarcal hegemónicos por replicar su terminología, lo hace desestabilizando esos mismos signos a través de la manera en que los articula en la práctica y en el uso cotidiano de los mitos. Bajo la apariencia guardada de que se trata de una religión tan buena como cualquier otra, de una familia tan legítima como cualquier otra, opera una corrosión de estos signos y su deslizamiento en la dirección de lo irreconocible y desestabilizador. Es por esto que, como he afirmado en otras ocasiones (1995b), se trata de una sociedad de "después de la caída", es decir, de después del Atlantic passage, una sociedad post-colonial: conoce su lugar, pero se desliza irónicamente hacia fuera de él.

En este contexto, existiría una divinidad que vendría a paradigmatizar todo lo dicho. Esta divinidad es el Exu, portero y sirviente de los orixas, pero sirviente poderoso, sin cuya colaboración ninguna puerta se abre, los caminos quedan impedidos, las relaciones interpersonales se destruyen. El Exu, que después de la diáspora crecería localmente hasta volverse dueño de un culto propio -el culto de Exu, una rama en franca expansión de la Umbanda- es la divinidad que simboliza esta mímesis progresiva de quien parodia las reglas del discurso y usa los nombres, pero no necesariamente los acata. Los Exus son blancos, beben champagne y se visten con capa y galera, pero son blancos de burdel, rufianes, espíritus de personajes de la noche; su elegancia es una elegancia parodiada, desestabilizadora, pues la exhiben en el ejercicio de su profesión de submundo, y la inscriben como un signo invertido en su mundo marginal.

\section{Campos, etnógráfos e interlocutores ocultos}

$\mathrm{Al}$ escribir sobre los Yoruba y formular sus modelos, Oyeronke Oyewumi y Lorand Matory se encuentran dialogando internamente con sus respectivas audiencias significativas, ambas anglosajonas; ella, llevada por un explícito e irreductible antagonismo; él, inspirado por un ánimo reformista conservador. Uno, queriendo el retorno de su antiguo mundo, y el otro, luchando para introducir los fragmentos, los despojos del viejo imperio "que no existe más" en el nuevo imperio, siempre ávido por anexaciones 
que puedan engrandecerlo. Como ellos, hasta un cierto punto, yo también me escondo detrás de mis datos, practicando ventrilocuismo con el nativo para pasar mi propio mensaje, que, sin embargo creo, no está enteramente distanciado del de ellos.

Por qué Oyeronke es tan enfática en su empeño por negar la existencia de una estructura de género - desde su punto de vista, una categoría occidental- entre los Yoruba pre-coloniales? Probablemente porque su mayor interlocutor en el discurso es aún el Occidente, ante el cual ella coloca las demandas de reconocimiento de su diferencia. Por qué el argumento de Lorand Matory enfatiza tanto el transvestitismo, disciplinando rigurosamente cualquier otra divergencia que el mundo Yoruba pueda contener en relación a la moralidad, el orden y la estructura de género occidental, y replegándolo, para esto, a una matriz heterosexual estricta, al mapa primario del dimorfismo jerárquico? Me parece que el subtexto del argumento del autor apunta para el problema fundamental del reclamo de derechos dentro - y no fuera- de los patrones de moralidad y el orden establecidos, la lucha del "buen ciudadano" quien, a pesar de alguna diferencia particular y superflua que pudiera ostentar en algún patrón de conducta-por ejemplo, en lo que respecta a su orientación sexual- nada más aspira que a un lugar apropiado para sí mismo, dentro de los límites estrechos y conservadores establecidos por la moralidad y la organización social y política dominante. A través de la etnografía, la primera trae su campo y lo enaltece como modelo purista e incontaminado para reclamar así el reconocimiento de su propia otredad; el otro lo trae como argumento de admisión en un mundo que no necesita ser transformado más que lo mínimamente necesario como para incorporar una diferencia "sartorial" - para utilizar sus propios términos.

Mi discurso tampoco carece de destinatario, y tal vez tenga más de uno. Por un lado, le digo a todos aquellos que disputan en el campo de la arena política establecida por los idiomas institucionales del estado, que los descendientes africanos en Brasil poseen un modo codificado, críptico, de criticar y de romper la fundación patriarcal de las instituciones brasileñas que los cercan. Pero que ese modo no es el de la dialéctica de las identidades políticas como el Occidente globalizado esperaría, sino un modo mucho más complejo y pleno de imprecisiones y ambivalencias.

La tradición africana se instaló en un nicho, en el interior de un contexto dominante al cual su discurso es referido, produciendo así un efecto de hibridismo. Este discurso reconoce, como dije, 
la existencia de un mundo circundante y hegemónico como otro, pero otro del cual la tradición, de una forma muy peculiar, forma parte. Ese otro mundo estaba ahí, dominante, cuando el culto se reconstituyó en el Nuevo Mundo, y continúa estando ahí, con fronteras reconocibles e intercambios a través de ellas. Por eso, su modo de ser es marginal, su identidad es una identidad en las márgenes, su fuerza es la fuerza del margen. El "pueblo del santo" nunca imaginó constituir un mundo separado, sino que siempre se vio como un pliegue hacia adentro en un mundo ya hegemonizado. La historia del Brasil moderno es representada, en las conversaciones y en la visitación diaria de la mitología, como un paisaje por donde se transita y no como el resultado de acciones y deliberaciones. Un paisaje que ya estaba allí, encontrado al llegar. Estar de paso por esa historia, en tránsito, no significa ser protagonista de esa historia. Esto determina una ambivalencia entre un sentimiento de estar allí pero no ser de allí.

En una ocasión, un prestigioso miembro de la tradición me dijo: "nuestra politica nunca es frontal, ostensiva". Leo, en esta afirmación, la constatación, por parte de un miembro representativo de la comunidad, que el discurso político afro-brasileño se formula siempre en la modalidad de la duplicidad de la voz, en el sentido ya examinado: mientras rinden culto a una familia formal en el panteón de dioses, la subvierten con las narrativas informales sobre el comportamiento sexual de sus miembros y las prácticas corrientes del ambiente social del culto. En su comentario irónico, la apariencia patriarcal y jerárquica del panteón mitológico encuentra su reverso. Al focalizar la ironía del comentario del culto a las instituciones patriarcales brasileñas, mi interpretación busca, de forma automática y no deliberada, profundizar la erosión subrepticia de la estructura convencional de género pero sin apegarme a ninguna otra articulación jerárquica que venga a substituir la que acabo de deconstruir. No es en nombre de otro orden, no es en nombre de un otro dialéctico. Éste me parece ser un procedimiento más radical.

Hibridez colonial no es un problema de genealogía o identidad entre dos culturas diferentes que pueda después resolverse como una cuestión de relativismo cultural [...] no es simplemente el contenido de los saberes negados. [...] Ver lo cultural no como una fuente de conflicto -en el sentido de culturas diferentes- sino como un efecto de prácticas discriminatorias -la producción de la diferenciación cultural como signo de autoridad -cambia su valor y sus reglas de reconocimiento. (Bhabha, 1994a:114, mi traducción) 
[...] la aprehensión de la identidad unificada, integrada y dialéctica, remite a la manera occidental de pensar la identidad -la identidad como imagen, que correponde a su dimensión metafórica, vertical, totémica. Mas la identidad es también dislocamiento, dada su dimensión metonímica [...] porque algo siempre falta a la presuposición de totalidad del signo. Es preciso recuperar su dimensión performática, aunque sea elíptica, aunque sea indefinida, más allá del discurso dialéctico que solamente repite al otro en su negación. (Pechincha, 2002:199)

En este sentido, mis interlocutores en sombra son los académicos y activistas norteamericanos, que con demasiada frecuencia reducen sus luchas por el reconocimiento de los derechos a la diferencia a meros recursos de admisión dentro del sistema, perdiendo de vista la necesidad de encuadrar y cuestionar el sistema mismo (Segato, 1998, 1999). Dialogo con ellos, y con la reificada noción de identidad política con la que muchas veces operan. Una noción que totaliza la condición de subalternidad, colocándola en posición especular y reactiva en relación a la condición de dominación, dentro de un paradigma de multiculturalismo estancado y anodino de lugares marcados en un mapa mundial - la "diversidad" pacata del multiculturalismo burgués se opone, en el pensamiento de Homi Bhabha, a la constante producción desestabilizadora de la "diferencia" (Bhabha, 1994b:34).

Si Oyeronke Oyewumi es la antagonista post-colonial, una nativista, alguien que afirma el principio de su Viejo Mundo como un otro enteramente respetable, en estado puro; Lorand Matory nos trae la noticia de una sociedad donde el transvestitismo, que aparentemente constituye una herejía moral para el occidente, no amenaza el orden establecido y, muy por el contrario, puede trabajar a favor del poder y ser funcional dentro de instituciones jerárquicas. Yo, ya me refiero y hago eco, con mi crítica, al discurso de una tradición que se perpetúa al lado y por debajo del discurso hegemónico del estado católico patriarcal brasileño y sus instituciones como un sub-discurso corrosivo, un coadyuvante incómodo, que con humor e ironía socava, desestabiliza y deconstruye el léxico de la dominación.

\section{NOTAS}

1. Lo que arregla, relativo a sastre - de costura o sutura.

2. La travesía del Atlántico en el tráfico de esclavos de África para el Nuevo Mundo. 


\section{Rita Segato}

\section{Referências Bibliográficas}

BAKHTIN, Mihail (1981). The dialogical imagination. Austin, Texas, The University of Texas Press.

BHABHA, Homi K. (1986). "Remembering Fanon. Introduction to the English edition of Black skin, white mask". London, Pluto Press.

_ (1994a). "Signs taken for wonders: Questions of ambivalence and authority under a tree outside Delhi, May 1817”. In H. Bhabha, The Location of culture. London e New York, Routledge.

_ (1994b). "The commitment to theory". In H. Bhabha, The Location of Culture. New York, Routledge

BIRMAN, Patricia (1995). "Fazer estilo criando gêneros”. Rio de Janeiro, UERJ/Relume-Dumará.

DIANTEILL, Erwan (2000) Des dieux et des signes. Initiation, écriture and divinitation dans les religions afro-cubaines. Paris, Éditions de l'École de Hautes Études en Sciences Sociales.

FRY, Peter (1977). "Mediunidade e sexualidade". Religião e Sociedade, 1.

_ (1986). "Male homosexuality and spirit possession in Brazil". Journal of Homosexuality, vol. 11, no 3-4.

LACAN, Jacques (1977). "The mirror stage as a formative of the function of the I". In J. Lacan, Écrits. A Selection. New York e London, W. W. Norton \& Company.

LANDES, Ruth (1940). "A cult matriarchate and male homosexuality". Journal of Abnormal and Social Psychology, no 25.

_ (1967). A cidade das mulheres. Rio de Janeiro, Civilização Brasileira.

LEACH, Edmund R. (1966). "Virgin birth". Proceedings of the Royal Anthropological Institute.

LESCOT, Anne \& MAGLOIRE, Laurence (2002). Des homes et dieux. Homosexuality and gay culture within the context of Haiti's vodou religion. Port-au- Prince, Haiti, video, 52 minutos.

MATORY, J. Lorand (1994). Sex and the Empire that is no more. Minneapolis, University of Minnesota Press.

OYEWUMI, Oyeronke (1997). The invention of women. Making an African sense of Western gender discourses. Minneapolis, University of Minnesota Press.

PECHINCHA, Mônica Theresa Soares (2002). Uma antropologia sem outro. O Brasil no discurso da antropologia nacional. Brasília, Tese de Doutorado, Programa de Pós-Graduação em Antropologia da Universidade de Brasília.

RIBEIRO, René (1969). "Personality and the psychosexual adjustment of Afro-Brazilian cult members". Journal de la Societé des Américanistes.

SAID, Edward... In Representar o Colonizado... (1997). Covering Islam. New York, Vintage Books.

SEGATO, Rita Laura (1986) "Inventando a natureza: família, sexo e gênero nos Xangôs de Recife”. Anuário Antropológico, 1985. Rio de Janeiro. (republicado in C. E. M. de Moura (ed.) 1989: Meu sinal está no teu corpo. São Paulo, EDICON/EDUSP, 1989; in R. L. Segato, Santos e Daimones: o politeismo a fro-brasileiro e a tradição arquetipal, Brasília, Editora da Universidade de Brasília, 1995; in C. E. M. de Moura (ed.), 
Candomblé: religiāo do corpo e da alma, Rio de Janeiro, Pallas, 2000; e in R. L. Segato, "Inventing Nature: family, sex and gender in the Xango cult", Acta Americana, 5(1), 1997, Estocolmo).

(1991). "Uma vocação de minoria: a expansão dos cultos afro-brasileiros na Argentina como processo de Re-Etnização”. Dados - Revista de Ciências Sociais, vol. 34, no 2.

(1995a). "Iemanjá e seus filhos: Fragmentos de um discurso político para compreender o Brasil". In Santos e Daimones: o politeísmo a fro-brasileiro e a tradição arquetipal. Brasília, Editora da Universidade de Brasília.

(1995b). "Cidadania: Por que não?. Estado e sociedade no Brasil à luz de um discurso religioso afro-brasileiro”. Dados - Revista de Ciências Sociais, vol. 38, nº 3 .

_ (1996). "Frontiers and margins: the untold story of the Afro-Brazilian religious expansion to Argentina and Uruguay". Critique of Anthropology, vol. 16, no 4.

_ (1998). "The color-blind subject of myth; or, where to find Africa in the nation". Annual Review of Anthropology 27.

(1999). "Identidades Políticas/Alteridades históricas. Una Crítica a las Certezas del Pluralismo Global”. Anuário Antropológico/97(republicado en forma abreviada para circulación en América Latina como "Identidades políticas y alteridades históricas. Una crítica a las certezas del pluralismo global". Nueva Sociedad, no 178 "Transnacionalismo y Transnacionalización”, marzo-abril 2002, pp.104-125.)

_ (2001). "Religião, vida carcerária e direitos humanos". In R. Novaes (org.), Direitos humanos. Temas e perspectivas. Rio de Janeiro, ABA/MAUAD/Fundação Ford.

WAFER, Jim (1991). The taste of blood. Spirit possession in Brazilian candomblé. Philadelphia, University of Pennsylvania Press. 